\title{
ON AN EXTREMUM PROBLEM
}

\author{
JOANNA MATULA
}

(Received 28 February 1984; revised 4 June 1985 and 28 February 1986)

\begin{abstract}
We consider an optimization problem in which the function being minimized is the sum of the integral functional and the full variation of control. For this problem, we prove the existence theorem, a necessary condition in an integral form and a local necessary condition in the case of monotonic controls.
\end{abstract}

\section{Introduction}

In the paper we deal with the problem of determining a minimum of the functional $\int_{0}^{1} f^{0}(t, x(t), u(t)) d t+\mathrm{V}_{0}^{1} u(t)$ under the constraints $\dot{x}(t)=$ $f(t, x(t), u(t)), x(0)=x_{0}, u \in U$, where $x$ is an absolutely continuous function on the segment $[0,1], x(t) \in R^{n}, x_{0}$ is a fixed point in $R^{n}$. $U$ stands for the set of all measurable vector-valued functions $u(t), t \in[0,1]$ with values in some compact set $M \subset R^{n}$. For the vector function $u$, we define the full variation $\bigvee_{0}^{1} u(t)$ as the sum of variations of its components.

The above extremal problem was formulated by E. S. Noussair in [8], Remark 3.3.

What distinguishes this problem from classical optimization problems is the form of the cost functional. Besides the differentiable part it also has a nondifferentiable term, namely the full variation of control on the interval $[0,1]$. This term can be interpreted as the cost of change of values of control function.

Section 1 of the paper contains a precise formulation of the problem and the proof of a theorem on the existence of an optimal solution. It is shown that the optimization problem considered is closely connected with the properties of controls with bounded variation. Optimization problems with such controls were

\footnotetext{
${ }^{1}$ Institute of Mathematics, Lodz University, u1. Stefana Banacha 22, 90-238 Lodz, Poland. (c) Copyright Australian Mathematical Society 1986, Serial-fee code 0334-2700/86
} 
investigated by B. E. Chuprun [2], N. N. Krasovskiy [6], S. Walczak [11], J. Sztajnic [10].

In Section 2 an integral necessary condition for the case of controls with bounded variation is proved. The proof is based on the Joffe-Tikhomirov extremum principle [4]. This principle was often applied to examining extremal problems in the spaces of real and complex functions (cf. [4], [7], [11]).

Section 3 of the paper deals with a local necessary condition for the existence of a solution in the case of monotonic controls. The form of this condition resembles the Euler-Lagrange equation; its proof is based on the results of [11]. To obtain the local necessary condition, we applied the local variational formula for monotonic functions with bounded variation. In particular, we used the wellknown variational formula of Golusin. Due to this formula, many functionals defined on special families of complex functions were estimated (see [3], [9]). In this paper the Golusin variational formula is used to investigate the optimization problems in the space of real functions.

\section{Formulation of the problem and an existence theorem}

Consider the following:

Problem 1. Determine the minimal value of the functional

$$
I(x, u)=\int_{0}^{1} f^{0}(t, x(t), u(t)) d t+\bigvee_{0}^{1} u(t)
$$

under the conditions

$$
\begin{gathered}
\dot{x}(t)=f(t, x(t), u(t)), \\
x(0)=x_{0}, \\
u \in U,
\end{gathered}
$$

where $x$ is an absolutely continuous function on the interval $[0,1], x(t) \in R^{n}, x_{0}$ is a fixed point in $R^{n}, U$ is a set of measurable vector functions $u=\left(u^{1}, \ldots, u^{r}\right)$ with values belonging to some compact set $M \subset R^{r}$ for $t \in[0,1]$. The set $U$ will be called a set of admissible controls, and $u \in U$-admissible controls.

The full variation of the vector function $u$ will be defined as

$$
\bigvee_{0}^{1} u(t)=\sum_{i=1}^{r} \mathfrak{V}_{0}^{1} u^{i}(t)
$$

Assume that the functions $f^{0}$ and $f$ satisfy the following conditions:

$1^{0} f^{0}(t, \cdot, \cdot)$ is lower semicontinuous on $R^{n} \times R^{r}$ with fixed $(x, u) \in R^{n} \times R^{r}$,

$2^{0}$ there exists a summable function $\mu(\cdot)$ such that $\mu(t) \leqslant f^{0}(t, x(t), u(t))$ for any process satisfying conditions (1.2)-(1.4) and for almost all $t \in[0,1]$, 
$3^{0} f$ is continuous with respect to $(x, u)$ and measurable with respect to $t$.

$4^{0}$ there exists a summable function $m(\cdot)$ such that $|f(t, x(t), u(t))| \leqslant m(t)$ for each process satisfying conditions (1.2)-(1.4) and for almost all $t \in[0,1]$.

Let $u_{0}(\cdot)$ be an arbitrary admissible control, $x_{0}(\cdot)$ its corresponding trajectory of system (1.2) with initial condition (1.3). Let us introduce the notation

$$
I\left(x_{0}, u_{0}\right)=K_{0}
$$

and

$$
U_{K}=\{u \in U \mid I(x, u) \leqslant K\} .
$$

$K$ is some constant greater than or equal to $K_{0}$, and $x(\cdot)$ a trajectory of system (1.2) with initial condition (1.3), corresponding to the control $u(\cdot)$.

The set $U_{K}$ is non-empty because, in particular, $u_{0} \in U_{K}$. Let $Q_{K}$ denote a set of solutions to equation (1.2) with initial condition (1.3) which correspond to the controls $u \in U_{K}$.

From the definition of the set $U_{K}$ it follows that

$$
\inf _{u \in U} I(x, u)=\inf _{u \in U_{K}} I(x, u) .
$$

Under assumptions $\left(1^{0}-4^{0}\right)$ one can easily show that $U_{K}$ is a set of vector functions whose values and variations are commonly bounded. So, while considering Problem 1, it is enough to restrict ourselves to the class of controls with variation bounded by a constant $K \geqslant K_{0}$.

Let $\left\{u_{n}(\cdot)\right\}, n=1,2, \ldots$, be a sequence of elements of the set $U_{K}$. By $x_{n}(\cdot)$ we denote the trajectories of system (1.2) with initial condition (1.3), that correspond to the controls $u_{n}(\cdot)$. If the sequence $\left\{u_{n}(\cdot)\right\}$ is pointwise convergent to some $\tilde{u}(\cdot) \in U_{K}$, then the sequence $\left\{x_{n}(\cdot)\right\}$ converges to $\tilde{x}(\cdot)$ uniformly on $[0, T]$, where $\tilde{x}(\cdot)$ is the solution of (1.2) with initial condition (1.3), corresponding to $\tilde{u}(\cdot)$ (see [1], Chap. 18). Making use of this fact, we prove

LEMMA 1.1. The functional

$$
J(x, u)=\int_{0}^{1} f^{\dot{\delta}}(t, x(t), u(t)) d t
$$

is sequentially lower semicontinuous on the set $Q_{K} \times U_{K}$ with respect to pointwise convergence of $(x(\cdot), u(\cdot))$.

Proof. Define $\bar{f}^{0}=\max \left(f^{0}, 0\right)$. Then it follows from Assumption $2^{0}$ that

$$
\tilde{f}^{0}(t, x(t), u(t))-\mu(t) \geqslant 0
$$

for any process $(x, u)$ that fulfills $(1.2)-(1.4)$ and for almost all $t \in[0,1]$.

Let $\left\{u_{n}(\cdot)\right\}, u_{n}(\cdot) \in U_{K}$ be a sequence pointwise convergent to $\tilde{u}(\cdot) \in U_{K}$. Now, let $v_{n}=\left(x_{n}, u_{n}\right)$ where, as usual, $x_{n}(\cdot)$ corresponds to $u_{n}(\cdot)$. Then, the sequence $\left\{v_{n}(\cdot)\right\}$ is pointwise convergent to $\tilde{v}=(\tilde{x}, \tilde{u}) \in Q_{K} \times U_{K}$. By the Fatou 
lemma [1], we have

$$
\begin{aligned}
& \int_{0}^{1} \lim _{n \rightarrow \infty} \inf \left[\bar{f}^{0}\left(t, v_{n}(t)\right)-\mu(t)\right] d t \\
& \quad \leqslant \lim _{n \rightarrow \infty} \inf \int_{0}^{1}\left[\bar{f}^{0}\left(t, v_{n}(t)\right)-\mu(t)\right] d t
\end{aligned}
$$

and hence,

$$
\int_{0}^{1} \lim _{n \rightarrow \infty} \inf \bar{f}^{0}\left(t, v_{n}(t)\right) d t \leqslant \lim _{n \rightarrow \infty} \inf \int_{0}^{1} f^{0}\left(t, v_{n}(t)\right) d t .
$$

Since $u_{n} \in U_{K}$, it follows that $I\left(x_{n}, u_{n}\right) \leqslant K$, i.e.

$$
J\left(v_{n}\right)=\int_{0}^{1} f^{0}\left(t, v_{n}(t)\right) d t \leqslant K<\infty .
$$

Let us recall that the functions $u \in U_{K}$ are commonly bounded with commonly bounded variations. The assertion now follows from the sufficient condition for semicontinuity given in [5].

LEMMA 1.2. The functional $T(u)=V_{0}^{1} u(t)$ is sequentially lower semicontinuous with respect to pointwise convergence of $u(\cdot)$.

Proof. Let $\tilde{u}(\cdot)=\lim _{n \rightarrow \infty} u_{n}(\cdot)$ on the interval $[0,1]$. It is enough to show that

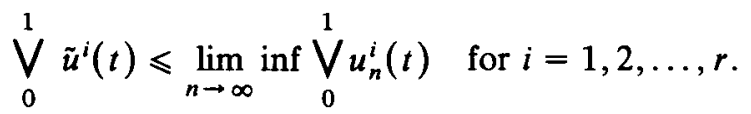

The index " $i$ " denotes the $i$-th component. If the right-hand side of (1.12) is equal to infinity, nothing is to be proved. So, suppose that it is finite and put

$$
\lim _{n \rightarrow \infty} \inf \bigvee_{0}^{1} u_{n}^{\prime}(t)=c \text {. }
$$

We take any $\varepsilon>0$ and let $0=t_{0}<t_{1}<\cdots<t_{m}=1$ be an arbitrary division of the interval $[0,1]$. Since

$$
\lim _{n \rightarrow \infty} \inf \bigvee_{0}^{1} u_{n}^{\prime}(t)<c+\varepsilon,
$$

there is a subsequence $\left\{u_{n}(\cdot)\right\}, n \in W \subset\{1,2, \ldots\}$ such that

$$
\bigvee_{0}^{1} u_{n}^{i}(t)<c+\varepsilon \text {. }
$$

This implies the inequality

$$
\sum_{j=1}^{m}\left|u_{n}^{i}\left(t_{j}\right)-u_{n}^{i}\left(t_{j-1}\right)\right|<c+\varepsilon
$$


Passing to the limit as $n \rightarrow \infty$, we get

$$
\sum_{j=1}^{m}\left|\tilde{u}^{i}\left(t_{j}\right)-\tilde{u}^{i}\left(t_{j-1}\right)\right| \leqslant c+\varepsilon .
$$

Taking the supremum of the left-hand sides of (1.14) over all possible divisions of $[0,1]$ we have

$$
\bigvee_{0}^{1} \tilde{u}^{\prime}(t) \leqslant c+\varepsilon
$$

Since $\varepsilon$ was arbitrary, we obtain

$$
\bigvee_{0}^{1} \tilde{u}^{i}(t) \leqslant c .
$$

But this means that inequality (1.12) is also true in the case when its right-hand side is finite. From definition (1.5) it follows that

$$
\varliminf_{0}^{1} \tilde{u}(t) \leqslant \lim _{n \rightarrow \infty} \inf \stackrel{1}{1}_{u_{n}}(t)
$$

which shows that the functional $T(u)$ is sequentially lower semicontinuous.

LEMMA 1.3. The set $U_{k}$ is sequentially compact with respect to pointwise convergence.

Proof. Consider a sequence $\left\{u_{n}(\cdot)\right\}$ where $u_{n}(\cdot) \in U_{K}, n=1,2, \ldots$ Then, $I\left(x_{n}, u_{n}\right) \leqslant K$ where $x_{n}(\cdot)$ is the solution of (1.2) with initial condition (1.3), corresponding to the control $u_{n}(\cdot)$. We now note that the functions $u_{n}(\cdot)$ and their variations are commonly bounded. Using Helly's choice principle (see [1], Chap. 15), we obtain the existence of a subsequence $\left\{u_{n}(\cdot)\right\}, n \in W \subset\{1,2, \ldots\}$, which is pointwise convergent on the interval $[0,1]$ to a function $\tilde{u}$ of bounded variation. To the sequence $\left\{u_{n}(\cdot)\right\}, n \in W \subset\{1,2, \ldots\}$, there corresponds a sequence $\left\{x_{n}(\cdot)\right\}, n \in W \subset\{1,2, \ldots\}$, of solutions of (1.2), (1.3). This sequence converges to a function $\tilde{x}(\cdot)$ uniformly on $[0,1]$, where $\tilde{x}(\cdot)$ is the solution of (1.2), (1.3), corresponding to the control $\tilde{u}(\cdot)$. Therefore, to prove the assertion, we are to show that $\tilde{u}(\cdot) \in U_{K}$, i.e. that $I(\tilde{x}, \tilde{u}) \leqslant K$.

Since $\left\{u_{n}(\cdot)\right\} \subset U_{K}$, it is clear that

$$
\int_{0}^{1} f^{0}\left(t, x_{n}(t), u_{n}(t)\right) d t+\bigvee_{0}^{1} u_{n}(t) \leqslant K
$$

Thus, we also have

$$
\lim _{n \rightarrow \infty} \inf \left(\int_{0}^{1} f^{0}\left(t, x_{n}(t), u_{n}(t)\right) d t+\bigvee_{0}^{1} u_{n}(t)\right) \leqslant K
$$


From the sequentially lower semicontinuity of the integral functional $\int_{0}^{1} f^{0}(t, x(t), u(t)) d t$ with respect to $(x, u)$ and the sequentially lower semicontinuity of the variation of $u(\cdot)$ we get

$$
I(\tilde{x}, \tilde{u})=\int_{0}^{1} f^{0}(t, \tilde{x}(t), \tilde{u}(t)) d t+\bigvee_{0}^{1} \tilde{u}(t) \leqslant K .
$$

Hence $\tilde{u}(\cdot) \in U_{K}$ and the proof is complete.

LEMMA 1.4. The set $Q_{K}$ is sequentially compact with respect to pointwise convergence.

Proof. Consider a mapping

$$
u(\cdot) \rightarrow x(\cdot)
$$

defined for $u(\cdot) \in U_{K}$, where $x(\cdot)$ denotes the corresponding trajectory of system (1.2) with condition (1.3). This mapping is sequentially continuous with respect to pointwise convergence. The image of the class $U_{K}$ of admissible controls, under this mapping, is the set $Q_{K}$. Now, the assertion follows from Lemma 1.3.

THEOREM 1.1. If the functions $f^{0}$ and f fulfill assumptions $1^{0}-4^{0}$, then Problem 1 has a solution.

Proof. We show that the functional $I(\cdot, \cdot)$ attains its infimum on the set $Q_{K} \times U_{K}$. Consider a sequence $\left\{\left(x_{n}(\cdot), u_{n}(\cdot)\right)\right\} \subset Q_{K} \times U_{K}, n=1,2, \ldots$ (here $x_{n}(\cdot)$ is, as usual, the trajectory that corresponds to the control $u_{n}(\cdot)$ and is subject to (1.2), (1.3)) such that

$$
I\left(x_{n}, u_{n}\right) \rightarrow \inf _{(x, u) \in Q_{K} \times U_{K}} I(x, u)=m .
$$

Since the set $Q_{K} \times U_{K}$ is sequentially compact we have that the sequence in question contains a subsequence $\left\{\left(x_{n}(\cdot), u_{n}(\cdot)\right)\right\}, n \in W \subset\{1,2, \ldots\}$ convergent to some $(\hat{x}(\cdot), \hat{u}(\cdot)) \in Q_{K} \times U_{K}$. Making use of sequentially lower semicontinuity of $I(x, u)$ we obtain

$$
m=\lim _{n \rightarrow \infty} I\left(x_{n}, u_{n}\right) \geqslant \lim _{n \rightarrow \infty} \inf I\left(x_{n}, u_{n}\right) \geqslant I(\hat{x}, \hat{u}) .
$$

On the other hand, $I(\hat{x}, \hat{u}) \geqslant m$ for $(\hat{x}, \hat{u}) \in Q_{K} \times U_{K}$. Hence $I(\hat{x}, \hat{u})=m$. Together with condition (1.6) this proves the theorem.

\section{The integral extremum principle}

On the grounds of the above considerations, it can be seen that we lose no generality by viewing Problem 1 in the class of controls with variation bounded 
by a constant $K_{0}=K-\int_{0}^{1} \mu(t) d t$, that is, by considering the following

Problem $1^{\prime}$. Determine the minimal value of the functional

$$
I(x, u)=\int_{0}^{1} f^{0}(t, x(t), u(t)) d t+\bigvee_{0}^{1} u(t)
$$

under the conditions

$$
\begin{gathered}
\dot{x}(t)=f(t, x(t), u(t)), \\
x(0)=x_{0}, \\
. u \in U_{K_{0}}
\end{gathered}
$$

where $U_{K_{0}}=\left\{u \in U \mid \mathrm{V}_{0}^{1} u(t) \leqslant K_{0}\right\}$.

In the sequel, we shall denote by $W_{1,1}^{n}(0,1)$ a space of functions $x(t) \in R^{n}$ absolutely continuous on the interval $[0,1]$, with norm $\|x\|=|x(0)|+\int_{0}^{1}|\dot{x}(t)| d t$. Denote by $\left(x_{*}, u_{*}\right)$ the solution to Problem $1^{\prime}$. To continue, let the following assumptions be satisfied:

$5^{0}$ The functions $f^{0}$ and $f$ do not depend explicitly on $t$, they are jointly continuous with respect to $x, u$, continuously differentiable with respect to $x$, differentiable with respect to $u$, and $f_{u}^{0}, f_{u}$ satisfy the Lipschitz condition with respect to $(x, u)$, that is,

$$
\left|f_{u}^{0}\left(x_{1}, u_{1}\right)-f_{u}^{0}\left(x_{2}, u_{2}\right)\right| \leqslant L\left(\left|x_{1}-x_{2}\right|+\left|u_{1}-u_{2}\right|\right)
$$

for some $L>0$ and, analogously, for $f_{u}$,

$6^{0}$ There exists a neighborhood $V \subset W_{1,1}^{n}(0,1)$ of the point $x_{*}$, such that, for any $x \in V$ and for each $t \in[0,1]$, the functions $f^{0}$ and $f$ satisfy the following convexity conditions: for any $u_{1} \in U_{K_{0}}, u_{2} \in U_{K_{0}}$ and for any $\lambda \in[0,1]$, there exists some $u \in U_{K_{0}}$ such that

$$
\begin{gathered}
f^{0}(x, u) \leqslant \lambda f^{0}\left(x, u_{1}\right)+(1-\lambda) f^{0}\left(x, u_{2}\right), \\
f(x, u)=\lambda f\left(x, u_{1}\right)+(1-\lambda) f\left(x, u_{2}\right) .
\end{gathered}
$$

Theorem 2.1. Let $\left(x_{*}, u_{*}\right)$ be the solution to Problem $1^{\prime}$. If assumptions $5^{0}$ and $6^{0}$ are satisfied, then there exists an absolutely continuous function $\Phi$ and a constant $\lambda_{0} \geqslant 0$, not identically zero, such that

$$
\frac{d \Phi}{d t}=-f_{x}^{T}\left(x_{*}, u_{*}\right) \Phi(t)+\lambda_{0} f^{0}\left(x_{*}, u_{*}\right), \quad \Phi(1)=0,
$$

and

$$
\begin{aligned}
\int_{0}^{1}- & H\left(\lambda_{0}, \Phi, x_{*}, u_{*}\right) d t+\lambda_{0}{\underset{0}{\bigvee}}_{0}^{1} u_{*}(t) \\
& =\min _{u \in U_{K}}\left[\int_{0}^{1}-H\left(\lambda_{0}, \Phi, x_{*}, u\right) d t+\lambda_{0}{\underset{0}{V}}_{0}^{1} u(t)\right]
\end{aligned}
$$


where the function $H$ is defined by the formula

$$
H\left(\lambda_{0}, \Phi, x, u\right)=(\Phi(t), f(x, u))-\lambda_{0} f^{0}(x, u) .
$$

Proof. Denote $X=W_{1,1}^{n}(0,1), Y=L_{1}^{n}(0,1) \times R^{n}$. Let $F: X \times U_{K_{0}} \rightarrow Y$ be an operator defined by the equality

$$
[F(x(\cdot), u(\cdot))](t)=\left(\dot{x}(t)-f(x(t), u(t)), x(0)-x_{0}\right) .
$$

Then Problem 1' can be formulated in the form:

Problem 1". Determine the minimal value of the functional

$$
I(x, u)=\int_{0}^{1} f^{0}(x(t), u(t)) d t+\bigvee_{0}^{1} u(t)
$$

under the conditions

$$
\begin{gathered}
F(x, u)=0, \\
u \in U_{K_{0}}
\end{gathered}
$$

It is easy to verify that, for Problem $1^{\prime \prime}$, the assumptions of the extremum principle for smooth-convex problems, given in [4] (\$1.1.3), are satisfied. So, it may be applied to this problem. Consequently, a necessary condition for the point $\left(x_{*}, u_{*}\right)$ to be the solution to Problem 1" is the existence of Lagrange multipliers $\lambda_{0} \geqslant 0, \lambda_{1}, \Phi$ not vanishing simultaneously and such that

$$
\begin{aligned}
& \lambda_{0} \int_{0}^{1}\left(f_{x}^{0}\left(x_{*}(t), u_{*}(t)\right), \bar{x}(t)\right) d t+\left(\lambda_{1}, \bar{x}(0)\right) \\
& \quad+\int_{0}^{1}\left(\Phi(t), \dot{x}(t)-f_{x}\left(x_{*}(t), u_{*}(t)\right) \bar{x}(t)\right) d t=0
\end{aligned}
$$

for any $\bar{x}(\cdot) \in W_{1,1}^{n}(0,1)$, and

$$
\begin{aligned}
\int_{0}^{1}\left[\lambda_{0} f^{0}\left(x_{*}(t), u_{*}(t)\right)-\left(\Phi(t), f\left(x_{*}(t), u_{*}(t)\right)\right)\right] d t+\lambda_{0} \bigvee_{0}^{1} u_{*}(t) \\
=\min _{u \in U_{K_{0}}}\left\{\int _ { 0 } ^ { 1 } \left[\lambda_{0} f^{0}\left(x_{*}(t), u(t)\right)\right.\right. \\
\left.\left.-\left(\Phi(t), f\left(x_{*}(t), u(t)\right)\right)\right] d t+\lambda_{0} \bigvee_{0}^{1} u(t)\right\} .
\end{aligned}
$$

By introducing the notation

$$
H\left(\lambda_{0}, \Phi, x, u\right)=(\Phi(t), f(x, u))-\lambda_{0} f^{0}(x, u),
$$

condition (2.8) can be written down in the form

$$
\int_{0}^{1}(\Phi(t), \dot{\bar{x}}(t)) d t+\int_{0}^{1}\left(H_{x}\left(\lambda_{0}, \Phi, x_{*}, u_{*}\right), \bar{x}(t)\right) d t+\left(\lambda_{1}, \bar{x}(0)\right)=0 .
$$


This, in turn, implies that

$$
\int_{0}^{1}\left(\Phi(t)-\int_{t}^{1} H_{x} d \tau, \dot{\bar{x}}(t)\right) d t+\left(-\int_{0}^{1} H_{x} d \tau+\lambda_{1}, \bar{x}(0)\right)=0
$$

This last equality holds for any $\bar{x}(\cdot) \in W_{1,1}^{n}(0,1)$. So, in particular, it holds for $\bar{x}(\cdot)$ such that $\bar{x}(0)=0$. Then $(2.12)$ will take the form

$$
\int_{0}^{1}\left(\Phi(t)-\int_{t}^{1} H_{x} d \tau, \dot{\bar{x}}(t)\right) d t=0 .
$$

Equality (2.13) holds for each $\bar{x}(\cdot) \in W_{1,1}^{n}(0,1)$ satisfying the condition $\bar{x}(0)=0$. Consequently, in particular, for $\bar{x}(\cdot)$ such that $\bar{x}(1)=0$. On the ground of the Du-Bois-Raymond lemma, (see [1], Chap. 2) we obtain that

$$
\Phi(t)-\int_{t}^{1} H_{x} d \tau=\text { const. }
$$

And so, $\Phi(t)$ is an absolutely continuous function which satisfies the differential equation

$$
\frac{d \Phi}{d t}=-f_{x}^{T}\left(x_{*}, u_{*}\right) \Phi(t)+\lambda_{0} f_{x}^{0}\left(x_{*}, u_{*}\right)
$$

where $f_{x}^{T}$ stands for the transpose of the matrix $f_{x}$. Let us again consider equality (2.13). Integrating the left-hand side of this equality by parts and making use of (2.15), we get

$$
\Phi(1) \bar{x}(1)=0 .
$$

Since (2.16) holds for any $\bar{x}(\cdot) \in W_{1,1}^{n}(0,1)$ satisfying the condition $\bar{x}(0)=0$, therefore

$$
\Phi(1)=0
$$

Writing condition (2.9) with the use of notation (2.10), we obtain the second condition of the proposition to the theorem. Theorem 2.1 has thus been proved.

\section{The local necessary condition in the class of monotone controls}

Let us now consider Problem $1^{\prime}$ in the class of controls which are monotone functions.

Problem 2. Determine the minimum of the functional

$$
I(x, u)=\int_{0}^{1} f^{0}(x(t), u(t)) d t+\bigvee_{0}^{1} u(t)
$$

under conditions

$$
\begin{gathered}
\dot{x}(t)=f(x(t), u(t)), \\
x(0)=x_{0}, \\
u \in \tilde{U}_{K_{0}},
\end{gathered}
$$


where, as previously, $x$ is an absolutely continuous function on the interval $[0,1]$, $x(t) \in R^{n}, x_{0}$ is a fixed point in $R^{n}$. $\tilde{U}_{K}$ is a set of monotonic vector functions (i.e., each component is a monotonic function) with values belonging to some compact set $M$ for $t \in[0,1]$ and with variation bounded by a constant $K_{0}$.

Assume that conditions $1^{0}-4^{0}$ given in Section 1 as well as conditions $5^{0}-6^{0}$ given in Section 2 are satisfied. All the above considerations are also true for the controls $u \in \tilde{U}_{K_{0}}$. So, analogously to before, for Problem 2 one can prove the existence theorem and the integral necessary condition in the form of theorem 2.1, where the set $\tilde{U}_{K_{0}}$ appears instead of $U_{K_{0}}$.

We shall now proceed to the proof of local necessary conditions for Problem 2. Apart from assumptions $1^{0}-2^{0}$ given in Section 1 , assume additionally that $7^{0} M$ is a perpendicular parallelepiped in $R^{r}$ of the form

$$
M=\left\{u \in R^{r} \mid u^{i} \in\left[\alpha_{i}, \beta_{i}\right], i=1,2, \ldots, r\right\},
$$

where $\alpha_{i}, \beta_{i}$ are some constants.

Denote $\bigvee_{0}^{1} u_{*}(t)=K^{*}$ and $\tilde{U}_{K^{*}}=\left\{u \in \tilde{U}_{K_{0}} \mid \bigvee_{0}^{1} u(t)=K^{*}\right\}$. For $u \in \tilde{U}_{K^{*}}$, the second part of the proposition to theorem 2.1 will take the form

$$
\int_{0}^{1}-H\left(\lambda_{0}, \Phi, x_{*}, u_{*}\right) d t=\min _{u \in \tilde{U}_{K^{*}}} \int_{0}^{1}-H\left(\lambda_{0}, \Phi, x_{*}, u\right) d t
$$

In the sequel, we need the following lemmas:

LEMMA 3.1. If an integral functional

$$
f(u)=\int_{0}^{1} a(t) u(t) d t=\sum_{i=1}^{r} \int_{0}^{1} a^{i}(t) u^{i}(t) d t
$$

is a functional supporting the set $U_{K_{0}}$ at the point $u_{0}$, then each of the functionals

$$
f^{i}(u)=\int_{0}^{1} a_{\imath}(t) u^{i}(t) d t
$$

is a functional supporting the set

$$
U_{K_{0}}^{i}=\left\{u^{i} \in L_{\infty} \mid \alpha_{i} \leqslant u^{i} \leqslant \beta_{i} \text { and } \stackrel{1}{\bigvee}_{0}^{i} \leqslant K_{0}^{i}\right\}
$$

at the point $u_{0}^{t}$, where $K_{0}^{i}=V_{0}^{1} u_{0}^{i}(t)$.

LEMMA 3.2. If a function $a:[0,1] \rightarrow R$ has a bounded variation, then the interval $[0,1]$ can be represented in the form

$$
[0,1]=N \cup E_{0} \cup \bigcup_{n=1}^{\infty} G_{n},
$$

where $N$ is a countable set, $E_{0}=\{t \in[0,1] \mid a(t)=0\}, G_{n}, n=1,2, \ldots$, are open intervals and $a(\cdot)$ is of constant sign on each of these intervals. 
The two lemmas were proved in paper [11].

Let $g_{0}$ be any monotone scalar function defined on the interval $[0,1]$, such that $g_{0}(t) \in[\alpha, \beta] \subset R$ and $\bigvee_{0}^{1} g_{0}=K$. Let $\left(t_{1}, t_{2}\right) \subset[0,1]$. Denote by $\mu$ one of the two numbers: $m, M$, where $m$ and $M$ stand for, respectively, the lower bound and the upper bound of the function $g_{0}$ on the interval $\left(t_{1}, t_{2}\right) \subset[0,1]$.

LEMMA 3.3. A function $g_{\lambda}$ defined by the formula

$$
g_{\lambda}(t)=\left\{\begin{array}{l}
g_{0}(t) \quad \text { for } t \notin\left(t_{1}, t_{2}\right), \\
(1-\lambda) g_{0}(t)+\lambda_{\mu} \quad \text { for } t \in\left(t_{1}, t_{2}\right)
\end{array}\right.
$$

for $\lambda \in[0,1]$ satisfies the following conditions:

$$
g_{\lambda}(t) \in[\alpha, \beta], \quad \bigvee_{0}^{1} g_{\lambda}(t)=K
$$

and is a monotone function, too.

Proof. If $t \notin\left(t_{1}, t_{2}\right)$, then $g_{\lambda}(t)=g_{0}(t)$ and $g_{\lambda}(t) \in[\alpha, \beta]$.

If $t \in\left(t_{1}, t_{2}\right)$, then $g_{\lambda}(t)=(1-\lambda) g_{0}(t)+\lambda \mu$ and thus

$$
\begin{gathered}
(1-\lambda) g_{0}(t)+\lambda \mu \geqslant(1-\lambda) m+\lambda m=m \geqslant \alpha, \\
(1-\lambda) g_{0}(t)+\lambda \mu \leqslant(1-\lambda) M+\lambda M=M \leqslant \beta .
\end{gathered}
$$

And so, $g_{\lambda}(t) \in[\alpha, \beta]$ for $t \in[0,1]$. Since $g_{0}$ is a monotone function, therefore $g_{\lambda}$ is monotone on the intervals $\left[0, t_{1}\right]$ and $\left[t_{2}, 1\right]$. It follows from inequalities (3.11) and (3.12) that it has this property on the entire interval $[0,1]$. Hence

$$
\bigvee_{0}^{1} g_{0}(t)=\left|g_{0}(0)-g_{\lambda}(1)\right|=\left|g_{0}(0)-g_{0}(1)\right|=\bigvee_{0}^{1} g_{0}(t)=K,
$$

and this concludes the proof of the lemma.

ThEOREM 3.1. If $\left(x_{*}, u_{*}\right)$ is the solution to Problem 2 and assumptions $5^{0}-7^{0}$ are satisfied, then there exist (not identically zero): an absolutely continuous function $\Phi$ satisfying differential equation (2.15) with terminal condition (2.17), a constant $\lambda_{0} \geqslant 0$ and a function $c=\left(c^{1}, c^{2}, \ldots, c^{r}\right)$ such that

a) $c^{i}$ is a constant function on each interval $G_{n}^{z}$,

b) $\left(f_{u}^{T}\left(x_{*}, u_{*}\right) \Phi(t)-\lambda_{0} f_{u}^{0}\left(x_{*}, u_{*}\right)\right)^{i}\left(c^{i}(t)-u_{*}^{i}(t)\right)=0$ for each $t \in[0,1]$, with the exception of at most a countable number of points.

Proof. Let $\left(t_{1}, t_{2}\right) \subset[0,1]$, and let

$$
u_{\lambda}(t)=\left\{\begin{array}{l}
u_{*}(t) \quad \text { for } t \notin\left(t_{1}, t_{2}\right) \\
u_{*}(t)+\lambda\left(\mu-u_{*}(t)\right) \text { for } t \in\left(t_{1}, t_{2}\right)
\end{array}\right.
$$


where $\mu=\left(\mu^{1}, \ldots, \mu^{r}\right), \mu^{i}$ is one of the two numbers $m^{i}, M^{\prime} . m^{i}$ and $M^{\prime}$ denote, respectively, the lower bound and the upper bound of the function $u_{*}^{i}(t)$ on the interval $\left(t_{1}, t_{2}\right), i=1,2, \ldots, r$. It follows from Lemma 3.3 that $u_{\lambda}(t) \in \tilde{U}_{K^{*}}$. On the ground of equality (3.5), we have

$$
\int_{0}^{1}-H\left(\lambda_{0}, \Phi, x_{*}, u_{*}\right) d t \leqslant \int_{0}^{1}-H\left(\lambda_{0}, \Phi, x_{*}, u_{\lambda}\right) d t
$$

Hence

$$
\int_{0}^{1}\left[H\left(\lambda_{0}, \Phi, x_{*}, u_{\lambda}\right)-H\left(\lambda_{0}, \Phi, x_{*}, u_{*}\right)\right] d t \leqslant 0 .
$$

By the definition (3.13) for $t \in\left[0, t_{1}\right] \cup\left[t_{2}, 1\right]$, the integrand is equal to zero. Consequently,

$$
\int_{t_{1}}^{t_{2}}\left[H\left(\lambda_{0}, \Phi, x_{*}, u_{*}+\lambda\left(\mu-u_{*}\right)\right)-H\left(\lambda_{0}, \Phi, x_{*}, u_{*}\right)\right] d t \leqslant 0 .
$$

By the mean value theorem,

$$
\int_{t_{1}}^{t_{2}} \lambda H_{u}\left(\lambda_{0}, \Phi, x_{*}, u_{*}\right)\left(\mu-u_{*}(t)\right) d t+o(\lambda) \leqslant 0 .
$$

Dividing both sides by $\lambda$ and passing with $\lambda$ to zero, we have

$$
\int_{t_{1}}^{t_{2}} H_{u}\left(\lambda_{0}, \Phi, x_{*}, u_{*}\right)\left(\mu-u_{*}(t)\right) d t \leqslant 0
$$

By the definition of (2.10),

$$
H_{u}\left(\lambda_{0}, \Phi, x_{*}, u_{*}\right)=f_{u}^{T}\left(x_{*}, u_{*}\right) \Phi(t)-\lambda_{0} f_{u}^{0}\left(x_{*}, u_{*}\right) .
$$

In view of the other part of assumption $5^{0}$, the function $H_{u}$ defined by formula (3.17) has a finite variation for $\Phi$ absolutely continuous. Consequently, the set of its points of discontinuity is countable.

According to Lemma 3.2, for each $i=1,2, \ldots, r$, the interval $[0,1]$ can be represented in the form

$$
[0,1]=N^{i} \cup E_{0}^{i} \cup \bigcup_{n=1}^{\infty} G_{n}^{i}
$$

where $N^{i}$ is a countable set, $E_{0}^{t}=\left\{t \in[0,1] \mid H_{u}^{i}=0\right\}, G_{n}^{t}, n=1,2, \ldots$, are open intervals and $H_{u}^{i}$ is of constant sign on each of these intervals.

Let us now consider inequality (3.16) once again. It follows from Lemma 3.1 that

$$
\int_{t_{1}}^{t_{2}} H_{u}^{i}\left(\lambda_{0}, \Phi, x_{*}, u_{*}\right)\left(\mu^{i}-u_{*}^{i}(t)\right) d t \leqslant 0, \quad i=1,2, \ldots, r .
$$

Suppose that, for $t \in\left(t_{1}, t_{2}\right), H_{u}^{i}>0$. Let us then put $\mu^{i}=\sup u_{*}^{i}(t)=c_{0}^{i}$, $t \in\left(t_{1}, t_{2}\right)$. If $u_{*}^{i} \neq c_{0}^{i}$ on the set of positive measure, then

$$
\int_{i_{1}}^{t_{2}} H_{u}^{i}\left(\lambda_{0}, \Phi, x_{*}, u_{*}\right)\left(c_{0}^{i}-u_{*}^{i}\right) d t>0,
$$

which contradicts inequality (3.19). 
If $H_{u}^{i}<0$ on the interval $\left(t_{1}, t_{2}\right)$, we would have to put $\mu^{t}=\inf u_{*}^{t}(t), t \in$ $\left(t_{1}, t_{2}\right)$.

The theorem has thus been proved.

EXAMPLE. Let $\tilde{U}_{K_{0}}$ be a set of monotone vector functions $u=\left(u^{1}, u^{2}\right)$ with values belonging to a rectangle

$$
M=\left\{\left(y^{1}, y^{2}\right) \in R^{2} \mid \alpha_{1} \leqslant y^{1} \leqslant \beta_{1}, \alpha_{2} \leqslant y^{2} \leqslant \beta_{2}\right\}
$$

for $t \in[0,1]$, and with variation bounded by a constant $K_{0}$.

Consider the following problem:

Find the minimum of the functional

$$
I(x, u)=\int_{0}^{1}(C x(t)+D u(t)) d t+\stackrel{\bigvee}{V}_{0}^{1} u(t)
$$

where the state of the controlled object is described by a differential equation of the form

$$
\begin{aligned}
& \dot{x}_{1}(t)=u^{2}(t), \\
& \dot{x}_{2}(t)=-x_{1}(t)+u^{1}(t),
\end{aligned}
$$

under the initial condition

$$
x(0)=x_{0}
$$

with

$$
u \in \tilde{U}_{K_{0}} .
$$

Let $C=(1,2), D=(1,1), x_{0}=(1,0)^{T}$. System (3.21) can be written down in the form

$$
\dot{x}=A x(t)+B u(t),
$$

where

$$
A=\left[\begin{array}{ll}
0 & 0 \\
-1 & 0
\end{array}\right], \quad B=\left[\begin{array}{ll}
0 & 0 \\
1 & 0
\end{array}\right]
$$

Conjugate equation (2.15) will then take the form

$$
\frac{d \Phi(t)}{d t}=-A^{T} \Phi(t)+\lambda_{0} C^{T}
$$

with the terminal condition

$$
\Phi(1)=0
$$

That is

$$
\begin{aligned}
& \dot{\Phi}^{1}=\Phi^{2}+\lambda_{0} \\
& \dot{\Phi}^{2}=2 \lambda_{0} .
\end{aligned}
$$


The solution to this system is of the form

$$
\begin{aligned}
& \Phi^{1}=\lambda_{0} t^{2}+C_{1} t+\lambda_{0} t+C_{2}, \\
& \Phi^{2}=2 \lambda_{0} t+C_{1} .
\end{aligned}
$$

Making use of terminal condition (3.23), we determine constants $C_{1}$ and $C_{2}$ :

$$
\begin{aligned}
& C_{1}=-2 \lambda_{0}, \\
& C_{2}=0 .
\end{aligned}
$$

Thus, the solution to this equation are functions,

$$
\begin{aligned}
& \Phi^{1}(t)=\lambda_{0} t^{2}-\lambda_{0} t, \\
& \Phi^{2}(t)=2 \lambda_{0} t-2 \lambda_{0} .
\end{aligned}
$$

It follows from these equalities that $\lambda_{0} \neq 0$. Indeed, if $\lambda_{0}=0$, then $\Phi^{1} \equiv 0$ and $\Phi^{2} \equiv 0$, which contradicts the extremum principle. Without loss of generality one may assume that $\lambda_{0}=1$.

Condition b) of theorem 3.1 will take the form

$$
\left(B^{T} \Phi(t)-\lambda_{0} D^{T}\right)^{i}\left(c^{i}(t)-u_{*}^{i}(t)\right)=0, \quad i=1,2 .
$$

The functions $\left(B^{T} \Phi(t)-\lambda_{0} D^{T}\right)^{i}, i=1,2$, which will be called switching functions, are defined by the equalities

$$
\begin{aligned}
& \left(B^{T} \Phi(t)-\lambda_{0} D^{T}\right)^{1}=2 t-3, \\
& \left(B^{T} \Phi(t)-\lambda_{0} D^{T}\right)^{2}=t^{2}-t-1 .
\end{aligned}
$$

The first component possesses in the interval $[0,1]$ at most one zero, the second-at most two. Consequently, the optimal control does exist and each of its components is piecewise constant. The first possesses at most one jump point, the other-at most two.

So, the component $u_{1}(\cdot)$ may be written in the form

$$
u_{1}(t)= \begin{cases}v_{1}, & t \in\left[0, \tau_{1}\right) \\ v_{2}, & t \in\left[\tau_{1}, 1\right],\end{cases}
$$

where $\alpha_{1} \leqslant v_{1} \leqslant v_{2} \leqslant \beta_{1}, 0 \leqslant \tau_{1} \leqslant 1, v_{1}, v_{2}, \tau_{1}$ are constants, or in the form

$$
u_{1}(t)= \begin{cases}\bar{v}_{1}, & t \in\left[0, \bar{\tau}_{1}\right) \\ \bar{v}_{2}, & t \in\left[\bar{\tau}_{1}, 1\right],\end{cases}
$$

where $\alpha_{1} \leqslant \bar{v}_{2} \leqslant \bar{v}_{1} \leqslant \beta_{1}, 0 \leqslant \bar{\tau}_{1} \leqslant 1, \bar{v}_{1}, \bar{v}_{2}, \bar{\tau}_{1}$ are constants.

On the other hand, $u_{2}(\cdot)$ may be of the form

$$
u_{2}(t)= \begin{cases}w_{1}, & t \in\left[0, t_{1}\right) \\ w_{2}, & t \in\left[t_{1}, t_{2}\right) \\ w_{3}, & t \in\left[t_{2}, 1\right],\end{cases}
$$


where $\alpha_{2} \neq w_{1} \leqslant w_{2} \leqslant w_{3} \leqslant \beta_{2}, 0 \leqslant t_{1} \leqslant t_{2} \leqslant 1, w_{1}, w_{2}, w_{3}, t_{1}, t_{2}$ are constants, or, analogously

$$
u_{2}(t)= \begin{cases}\bar{w}_{1}, & t \in\left[0, \bar{t}_{1}\right) \\ \bar{w}_{2}, & t \in\left[\bar{t}_{1}, \bar{t}_{2}\right) \\ \bar{w}_{3}, & t \in\left[\bar{t}_{2}, 1\right],\end{cases}
$$

where $\alpha_{2} \leqslant \bar{w}_{3} \leqslant \bar{w}_{2} \leqslant \bar{w}_{1} \leqslant \beta_{2}, 0 \leqslant \bar{t}_{1} \leqslant \bar{t}_{2} \leqslant 1, \bar{w}_{1}, \bar{w}_{2}, \bar{w}_{3}, \bar{t}_{1}, \bar{t}_{2}$ are constants. Hence, we finally conclude that four cases of forms of $u_{1}(\cdot)$ and $u_{2}(\cdot)$ are possible.

Let us consider the case when $u_{1}(\cdot)$ is of form (3.25) and $u_{2}(\cdot)$ of form (3.27). We take the case when $\tau_{1} \leqslant t_{1}$.

For $t \in\left[0, \tau_{1}\right)$, system (3.21) has the form

$$
\left\{\begin{array}{l}
\dot{x}_{1}(t)=w_{1}^{2} \\
\dot{x}_{2}(t)=-x_{1}(t)+v_{1}
\end{array}\right.
$$

with the initial condition $x(0)=(1,0)^{T}$.

Solving this system, we get

$$
\left\{\begin{array}{l}
x_{1}(t)=w_{1}^{2} t+1 \\
x_{2}(t)=-\frac{1}{2} w_{1}^{2} t^{2}+\left(v_{1}-1\right) t \quad \text { for } t \in\left[0, \tau_{1}\right) .
\end{array}\right.
$$

For $t \in\left[\tau_{1}, t_{1}\right)$, system (3.21) becomes

$$
\left\{\begin{array}{l}
\dot{x}_{1}(t)=w_{1}^{2} \\
\dot{x}_{2}(t)=-x_{1}(t)+v_{2}
\end{array}\right.
$$

with the initial condition

$$
x\left(\tau_{1}\right)=\left(w_{1}^{2} \tau_{1}+1,-\frac{1}{2} w_{1}^{2} \tau_{1}^{2}+\left(v_{1}-1\right) \tau_{1}\right)^{T},
$$

and it has the following solution:

$$
\left\{\begin{array}{l}
x_{1}(t)=w_{1}^{2} t+1 \\
x_{2}(t)=-\frac{1}{2} w_{1}^{2} t^{2}+\left(v_{2}-1\right) t+\left(v_{1}-v_{2}\right) \tau_{1}, \quad \text { for } t \in\left[\tau_{1}, t_{1}\right) .
\end{array}\right.
$$

If $t \in\left[t_{1}, t_{2}\right)$ system (3.21) has the form

$$
\left\{\begin{array}{l}
\dot{x}_{1}(t)=w_{2}^{2} \\
\dot{x}_{2}(t)=-x_{1}(t)+v_{2}
\end{array}\right.
$$

with the initial condition

$$
x\left(t_{1}\right)=\left(w_{1}^{2} t_{1}+1,-\frac{1}{2} w_{1}^{2} t_{1}^{2}+\left(v_{2}-1\right) t_{1}+\left(v_{1}-v_{2}\right) \tau_{1}\right)^{T} .
$$


Having solved the above system, we get, for $t \in\left[t_{1}, t_{2}\right)$,

$$
\left\{\begin{array}{l}
x_{1}(t)=w_{2}^{2} t+\left(w_{1}^{2}-w_{2}^{2}\right) t_{1}+1 \\
x_{2}(t)=-\frac{1}{2} w_{2}^{2} t^{2}+\left[\left(w_{2}^{2}-w_{1}^{2}\right) t_{1}-1+v_{2}\right] t-\frac{1}{2}\left(w_{2}^{2}-w_{1}^{2}\right) t_{1}^{2}+\left(v_{1}-v_{2}\right) \tau_{1} .
\end{array}\right.
$$

Similarly, for $t \in\left[t_{2}, 1\right],(3.21)$ becomes

$$
\left\{\begin{array}{l}
\dot{x}_{1}(t)=w_{3}^{2}, \\
\dot{x}_{2}(t)=-x_{1}(t)+v_{2}
\end{array}\right.
$$

with

$$
\begin{array}{r}
x\left(t_{2}\right)=\left(w_{2}^{2} t_{2}+\left(w_{1}^{2}-w_{2}^{2}\right) t_{1}+1,-\frac{1}{2} w_{2}^{2} t_{2}^{2}+\left[\left(w_{2}^{2}-w_{1}^{2}\right) t_{1}-1+v_{2}\right] t_{2}\right. \\
\left.-\frac{1}{2}\left(w_{2}^{2}-w_{1}^{2}\right) t_{1}^{2}+\left(v_{1}-v_{2}\right) \tau_{1}\right)^{T},
\end{array}
$$

and its solution is determined by the formulae

$$
\left\{\begin{aligned}
x_{1}(t)= & w_{3}^{2} t+\left(w_{2}^{2}-w_{3}^{2}\right) t_{2}+\left(w_{1}^{2}-w_{2}^{2}\right) t_{1}+1 \\
x_{2}(t)= & -\frac{1}{2} w_{3}^{2} t^{2}+\left[\left(w_{3}^{2}-w_{2}^{2}\right) t_{2}+\left(w_{2}^{2}-w_{1}^{2}\right) t_{1}-1+v_{2}\right] t \\
& -\frac{1}{2}\left(w_{3}^{2}-w_{2}^{2}\right) t_{2}^{2}-\frac{1}{2}\left(w_{2}^{2}-w_{1}^{2}\right) t_{1}^{2}+\left(v_{1}-v_{2}\right) \tau_{1} .
\end{aligned}\right.
$$

Now, by putting in (3.20) $C=(1,2), D=(1,1)$ and using (3.25), (3.27), functional (3.20) may be written down in the form

$$
\begin{aligned}
I(x, u)= & \int_{0}^{\tau_{1}}\left[x_{1}(t)+2 x_{2}(t)+\left(v_{1}+w_{1}\right)\right] d t \\
& +\int_{\tau_{1}}^{t_{1}}\left[x_{1}(t)+2 x_{2}(t)+\left(v_{2}+w_{1}\right)\right] d t \\
& +\int_{t_{1}}^{t_{2}}\left[x_{1}(t)+2 x_{2}(t)+\left(v_{2}+w_{2}\right)\right] d t \\
& +\int_{t_{2}}^{1}\left[x_{1}(t)+2 x_{2}(t)+\left(v_{2}+w_{3}\right)\right] d t \\
& +\left(v_{2}-v_{1}\right)+\left(w_{3}-w_{1}\right) .
\end{aligned}
$$

Making use of (3.29)-(3.32) while computing the above integrals, we get

$$
I(x, u)=g\left(\tau_{1}, t_{1}, t_{2}, v_{1}, v_{2}, w_{1}, w_{2}, w_{3}\right),
$$

where $g$ is a function of the third degree with respect to $\tau_{1}, t_{1}, t_{2}$ and of the second degree with respect to $v_{1}, v_{2}, w_{1}, w_{2}, w_{3}$. 
In this way our problem, in the case considered, is reduced to the following mathematical programming problem:

"Determine the constants $\tau_{1}, t_{1}, t_{2}, v_{1}, v_{2}, w_{1}, w_{2}, w_{3}$ that give the minimum of the function

$$
g\left(\tau_{1}, t_{1}, t_{2}, v_{1}, v_{2}, w_{1}, w_{2}, w_{3}\right)
$$

subject to the linear constraints

$$
\begin{aligned}
& \alpha_{1} \leqslant v_{1} \leqslant v_{2} \leqslant \beta_{1}, \quad 0 \leqslant \tau_{1} \leqslant 1, \\
& \alpha_{2} \leqslant w_{1} \leqslant w_{2} \leqslant w_{3} \leqslant \beta_{2}, \quad 0 \leqslant t_{1} \leqslant t_{2} \leqslant 1 .
\end{aligned}
$$

Considering the remaining three cases in a similar way, we can finally conclude that the problem considered in the Example is reduced to some mathematical programming problem under linear constraints.

\section{References}

[1] L. Cesari, Optimization-theory and applications, (Springer-Verlag, New York, 1983).

[2] B. E. Chuprun, "Zadachi s ogranicheniyem na izmeneniye upravleniya", Avtomat $i$ Telemekh. 3 (1975), 24-37.

[3] G. M. Golusin, Geometricheskaja teorıya funktsii kompleksnogo peremennogo (Nauka, Moskva, 1966).

[4] A. D. Ioffe and V. M. Tikhomirov, Theory of extremal problems, (North-Holland, New York, 1979).

[5] A. D. Ioffe, "On Lower Semicontinuity of Integral Functionals", SIAM J. Control Optim. 15 (1977).

[6] N. N. Krasovskiy, Teoriya upravleniya dvizheniem, (Nauka, Moskva, 1968).

[7] L. Mikolajczyk and S. Walczak, "Application of the extremum principle to investigating certain extremal problems", Trans. Amer. Math. Soc. 259 (1980).

[8] E. S. Noussair, "On the existence of piecewise continuous optimal controls," J. Austral. Math. Soc. Ser. B 20 (1977), 31-37.

[9] J. Pfaltzgraff and B. Pinchuk, "A Variational Method for Classes of Meromorphic Functions", J. Analyse Math. (1971), 101-150.

[10] J. Sztajnic, "Necessary conditions for existence of extremum for controls with bounded variation", Bull. Soc. Sci. Lett. Lódź, XXVIII, 12 (1978).

[11] S. Walczak, "Euler-Lagrange's conditions for controls with bounded variation", Control Cybernet. 8 (1979). 